\title{
Chorioamnionitis and funisitis due to Corynebacterium kutscheri
}

\author{
W. F. FITTER, D. J. DE SA, AND H. RICHARDSON \\ Department of Pathology, McMaster University Medical Centre, Hamilton, Ontario, Canada
}

SUMMARY When isolated from the female genital tract, diphtheroids are usually regarded as commensal organisms. Corynebacterium kutscheri however is a pathogen in laboratory rodents. We report a case in which $C$. kutscheri was isolated as a pure culture from the umbilical cord and from other surface sites in an infant. Histological examination of the cord and membranes demonstrated the organisms within these fetal tissues. The organisms evoked a fetal cellular response. The importance of recognising commensals as potential pathogens in states of altered host resistance is stressed.

Chorioamnionitis (inflammation of fetal membranes and the fetal surface of the placenta) and funisitis (inflammation of the umbilical cord) are often caused by ascending infections from the lower genital tract, and organisms that are regarded as normal commensals in this area are generally responsible. Although diphtheroids are part of the normal flora of the genital tract (Leppäluoto, 1974; Galask et al., 1976) and have been isolated from the placental fetal surface and the fetal membranes (Benirschke and Driscoll, 1967), their pathogenicity is uncertain. As Benirschke and Driscoll (1967) stated, 'Results of cultures per se, were inconclusive. Doubtless this was because most of the placentas had traversed the lower genital tract prior to examination, and because the cultures were then taken from the exposed amniotic surface. However, taken in conjunction with other data, bacteriological cultures become very useful'.

It is worth re-emphasising that an 'infection' is usually defined as the invasion and multiplication of an organism within living tissues of the host (Walter and Israel, 1974). This report describes what we believe may be the first case of infection of the fetal membranes and cord in an infant due to a Corynebacterium species.

\section{Case report}

A baby girl was born prematurely at 26 weeks' gestation to a 42-year-old grand multiparous

\footnotetext{
Department of Pathology, McMaster University Medical Centre, Hamilton, Ontario

W. F. FITTER, chief resident

D. J. DE SA, staff pathologist

H. RICHARDSON, staff microbiologist
}

woman. Labour had started 24 hours earlier and was associated with leakage of amniotic fluid. Further rupture of membranes with discharge of amniotic fluid occurred immediately before birth. The baby was delivered vaginally as a vertex presentation. Apart from the sustained onset of premature labour, the pregnancy had been uneventful.

The infant weighed $980 \mathrm{~g}$ with an Apgar score of 3 after one minute and required assisted ventilation. The infant had periodic apnoea during the first 24 hours after delivery and was hypothermic.

Laboratory investigations were undertaken to exclude the possibility of sepsis and the infant was noted to have a WBC of $17 \cdot 4 \times 10^{9} / 1$ with a pronounced 'shift-to-the-left', and $20 \%$ of the white cells were 'band cells'. The cord haemoglobin was $16.6 \mathrm{~g} / \mathrm{dl}$. No growth was obtained from a blood culture or cerebrospinal fluid specimens. Cultures from the umbilical cord, fetal membranes, rectum, nose, throat, eye, and external ear gave a pure growth of an aerobic Gram-positive bacillus.

Bacteriological studies. The rods were slender and clubbed and some had pointed ends. Staining was irregular and metachromatic granules were present. When cultured on blood agar plates the organisms were greyish in colour with an irregular edge, and measured about $2 \mathrm{~mm}$ after overnight incubation. There was no evidence of haemolysis. The organisms fermented sucrose, and produced urease. Nitrate was not reduced and gelatinase was not produced. The organism was identified as C. kutscheri, and this was confirmed by the external reference centres at the Hospital for Sick children, Toronto, and the Central Public Health Laboratory, Toronto.

Chest $x$-rays showed bilateral infiltrates which 
increased during the next 24 hours, and these changes were considered to be consistent with a pneumonitis. After treatment with ampicillin and gentamicin there was considerable improvement in the infant's body temperature and a slow resolution of the pulmonary infiltrates was seen on subsequent $x$-rays. The abnormalities in the blood film disappeared gradually over a week. No organism was recovered at any time from the tracheal aspirates, however, and there was no firm microbiological confirmation of a C. kutscheri pneumonitis.

Pathological studies. The placenta, membranes, and cord were available for pathological examination. The placenta weighed $300 \mathrm{~g}$ with obvious discoloration of the fetal membranes by multiple, minute, greyish-brown plaques on the amnion. Similar minute plaques, $0 \cdot 2-0.5 \mathrm{~mm}$ in diameter, were identified on the surface of the umbilical cord (Fig. 1). Microscopical examination showed a florid chorioamnionitis and funisitis (Fig. 2). The plaques consisted of minute central necrotic foci with aggregates of mononuclear cells forming a boundary around invading colonies of Gram-positive bacilli (Fig. 3). In some of the organisms metachromatic granules were present. The organisms penetrated into and proliferated within the subamniotic mesenchyme of the fetal surface of the placenta, and Wharton's jelly in the umbilical cord. Fetal inflammatory cells could be seen migrating through the walls of the umbilical vessels in the cord and on the fetal surface of the placenta towards the invading

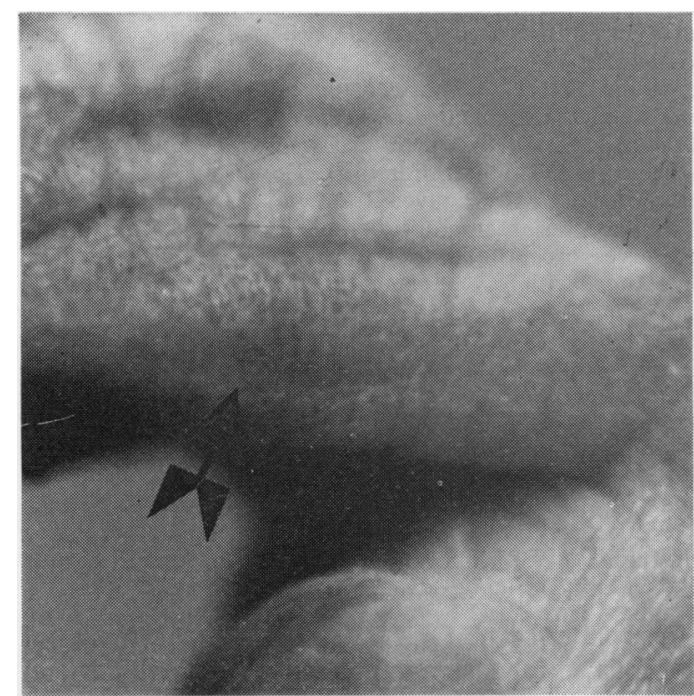

Fig. 1 Close up view of surface of umbilical cord of infant showing the multiple small greyish-brown plaques (arrow) on its surface (approximately $\times 2$ magnification).

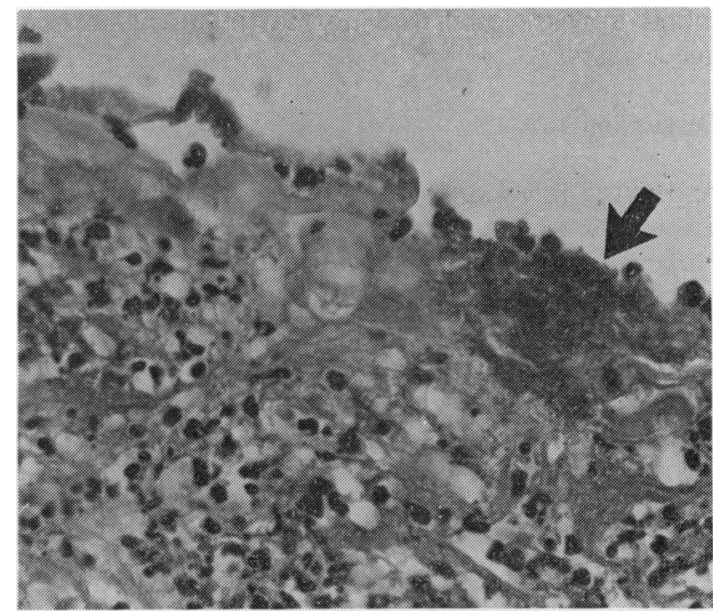

Fig. 2 Microscopical section through umbilical cord showing the proliferating colony of diphtheroids (arrow) and the cellular inflammatory response in Wharton's jelly. Papanicolaou's stain, $\times 800$.

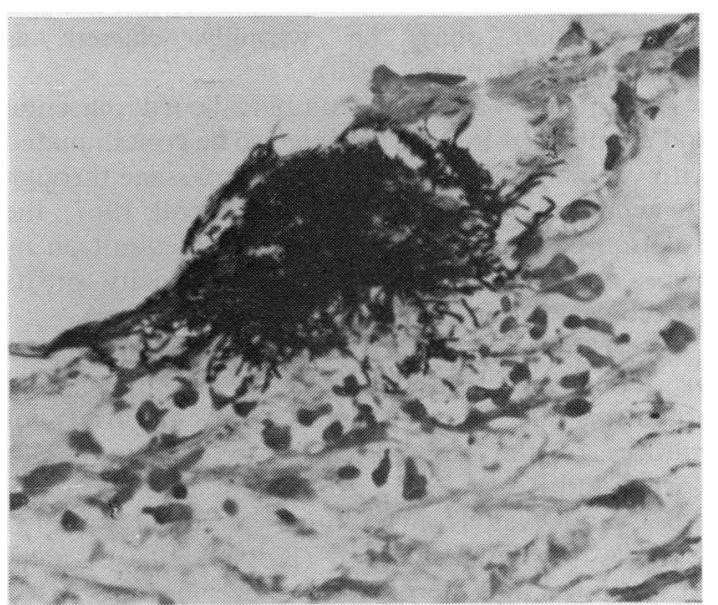

Fig. 3 Gram stain of one of the plaques seen in Fig. 1, showing the proliferating Gram-positive rods within Wharton's jelly. Note also the early cellular response to the organism. Gram stain, $\times 800$.

organisms. There was no evidence of inflammation of chorionic villi. The histological appearances were of a chorioamnionitis and funisitis, in which it was possible to demonstrate invading and proliferating organisms, morphologically compatible with those isolated from the surface of the cord and membranes, evoking a fetal inflammatory cellular reaction.

Follow-up. This baby has been followed up repeatedly after being discharged at 11 weeks (postnatal age). Weight gain has been slow, but consistent, and the 
infant appears otherwise normal in all respects at age 2 years.

\section{Discussion}

Ascending infections of the female genital tract are associated most commonly with isolates of Escherichia coli, anaerobic cocci, and Bacteroides species (Galask et al., 1976), all flora that normally inhabit the female genital tract (Tashjian et al., 1976).

C. kutscheri (formerly classified as Corynebacterium murium) has been known to be a cause of pseudotuberculous, granulomatous infection in laboratory rodents (Fauve et al., 1964; Pierce-Chase et al., 1964). However, like other diphtheroids, it is regarded as a normal commensal when isolated from the female genital tract (Galask et al., 1976; Tashjian et al., 1976). Diphtheroids are, of course, recognised often on routine cervical cytological examination in asymptomatic women (Leppäluoto, 1974). In fact, diphtheroids are such common inhabitants of the vagina that they can prove a source of diagnostic confusion as pathogenic Listeria monocytogenes may be wrongly classed as diphtheroids (Morison, 1970).

As the fetal skin surface, umbilical cord, placenta, and membranes may be expected to be contaminated with vaginal organisms during their passage through the genital tract (Benirschke and Driscoll, 1967), the isolation of a commensal organism does not on its own constitute proof of its pathogenicity or its aetiological role in producing chorioamnionitis.

As has been shown by many workers (summarised by Benirschke and Driscoll, 1967) diphtheroid organisms are often isolated from a variety of cultures of the vaginal cavity (Weinstein, 1938; Torrey and Reese, 1945), amniotic surface (Driscoll, 1967), and even from cultures of blood sampled through the umbilical cord (Kobak, 1930). In all these reports diphtheroids have never been reported in a pure culture, and in no instance was it suggested they were pathogenic.

In our patient however, we have been fortunate in demonstrating the invasion of fetal tissues by an organism that is often regarded as a banal commensal, and have been able to show it inducing an inflammatory response from the fetus. We were successful, furthermore in isolating it as a pure uncontaminated culture. It is possible for us to be confident, therefore, in identifying $C$. kutscheri as the causal agent in our particular infant's chorioamnionitis and funisitis. While we believe that this may be the first case where this organism has been implicated in an infection in humans, it is worth mentioning that the circumstances were fortuitous. It is probable, therefore, that other cases of $C$. kutscheri chorioamnionitis have occurred, but not been recognised or reported.

We believe that in our patient there were sufficient clinical grounds for suspecting a pneumonitis even though we were unable to obtain firm microbiological proof. The improvement in clinical status, in parallel with the changes in the $x$-rays of the chest and amelioration of the haematological abnormalities, after appropriate treatment with antibiotics does not mitigate against this view.

This case indicates the importance of recognising the possible role of commensals as potential pathogens when host resistance may be altered, as in extreme immaturity and in the presence of leaking amniotic fluid, with a breakdown in the natural barriers to infection. In such a situation the isolation of pure cultures, even of commensal microorganisms, demands careful assessment and close co-operation between microbiologist and pathologist.

\section{References}

Benirschke, K., and Driscoll, S. G. (1967). The Pathology of the Human Placenta. pp. 244-246. Springer: New York.

Driscoll, S. G. (1967). Fetal infections in man, In Comparative Aspects of Reproductive Failure, pp. 279-295. Edited by K. Benirschke. Springer: New York.

Fauve, R. M., Pierce-Chase, C. H., and Dubos, R. (1964). Corynebacterial pseudotuberculosis in mice. II. Activation of natural and experimental latent infections. Journal of Experimental Medicine, 120, 283-304.

Galask, R. P., Larsen, B., and Ohm, M. J. (1976). Vaginal flora and its role in disease entities. Clinical Obstetrics and Gynecology, 19, 61-81.

Kobak, A. J. (1930). Fetal bacteremia: a contribution to the mechanism of intrauterine infection and to the pathogenesis of placentitis. American Journal of Obstetrics and Gynecology, 19, 299-316.

Leppäluoto, P. (1974). The occurrence of vaginal diphtheroids in Papanicolaou smears. Acta cytologica, 18, 362-366.

Morison, J. E. (1970). Foetal and Neonatal Pathology, third edition pp. 539-540. Butterworth: London.

Pierce-Chase, C. H., Fauve, R. M., and Dubos, R. (1964). Corynebacterial pseudotuberculosis in mice. I. Comparative susceptibility of mouse strains to experimental infection with Corynebacterium kutscheri. Journal of Experimental Medicine, 120, 267-281.

Tashjian, J. H., Coulam, C. B., and Washington, J. A., II. (1976). Vaginal flora in asymptomatic women. Mayo Clinic Proceedings, 51, 557-561.

Torrey, J. C., and Reese, M. K. (1945). Initial aerobic flora of newborn infants. American Journal of Diseases of Children, 69, 208-214.

Walter, J. B., and Israel, M. S. (1974). General Pathology, fourth edition, p. 84. Churchill Livingstone: Edinburgh.

Weinstein, L. (1938). The bacterial flora of the human vagina. Yale Journal of Biology and Medicine, 10, 247-260.

Correspondence to Dr D. J. de Sa, Department of Pathology, McMaster University Medical Centre, 1200 Main Street West, Hamilton, Ontario, Canada L8S 4 J9.

Received 2 January 1979 